explaining the differences in stability constants of the complexes and here also an electrondonating or -withdrawing group at $p$-position of aniline seems to influence substantially the complex formation between $\mathrm{Fe}^{3+}$ and aryl $\beta$-ketoimines.

Aknowledgement The authors thank to Mr. M. Hojo for his helpful contribution to this study and to Mr. Y. Itatani and Miss. Y. Arano of this Faculty for elemental analyses.

\title{
Effect of Pyrazolone Derivatives on the Critical Micelle Concentration of Ionic Surfactants
}

\author{
Yasunori Morimoto, Tokiko IzUmi, ${ }^{\left.1 a_{1}\right)}$ and Takaichi Arita ${ }^{1 b}$ \\ Faculty of Pharmaceutical Sciences, Josai University ${ }^{\mathbf{1} a)}$ and Faculty of \\ Pharmaceutical Sciences, Hokkaido University ${ }^{1 b}$ )
}

(Received April 4, 1978)

\begin{abstract}
Effect of pyrazolone derivatives on the critical micelle concentration (cmc) of surfactants was investigated by using ionic surfactants which were sodium dodecyl sulfate (SDS) and dodecyl N-dimethylethylammonium bromide (DDAB). It is shown that antipyrine and 4-aminoantipyrine which are very water soluble is in breaking up micelles of SDS and DDAB. However, addition of phenylbutazone which is very slightly soluble in water results in increase in stability of the micelles. It is presumed from these results that the affinity of these additive for water plays an important role in stability of the micelles.
\end{abstract}

Keywords_hydrophobic interaction; critical micelle concentration; micelle stability; ionic surfactant; pyrazolone derivertives

The preferential association of non-polar groups with each other rather than with the surrounding water molecules is generally referred to as hydrophobic interactions or, less precisely, as hydrophobic or lyophobic bonding. ${ }^{2}$ ) Hydrophobic interactions are responsible to a large degree for stabilization of the secondary and tertiary structures of proteins in aqueous solutions, and protein denaturation is a consequence, at least in part, of the weakening of the hydrophobic interactions of the native proteins by denaturing agents such as urea and guanidium salts. ${ }^{3)}$ Generally denaturing agents are very water soluble and their efficacy has been ascribed to the breaking of protein-protein hydrogen bonds and preferential formation of protein-denaturants hydrogen bonds. And also it was suggested that the urea-induced increase of the drug-protein binding may be related to a disorganization of the protein structures probably caused by a reduction of the intramolecular hydrophobic force. ${ }^{3 a, 4)}$

However, although the drug-protein binding have been widely investigated, ${ }^{5)}$ attempts to analyze how the intramolecular hydrophobic forces are influenced by various drugs have

1) I.ocation: a) 1-1 Keyakidar, Sakado, Saitama, 350-02, Japan; b) Nishi 6-chome, Kita-12-jo, Kita-ku, Sapporo, 060, Japan.

2) W. Kauzmann, Adv. Protein Chem., 14, 1 (1959); G. Nemethy, Angew. Chem. Int. Ed. Engl., 6, 195 (1967).

3) a) P.L. Whitney and.C. Tanfords, J. Biol. Chem., 237, PC 1735 (1962); b) J.A. Gordon, Biochemistry, 11,1862 (1972).

4) J.D. Coombes, E. Katchalski, and P. Doty, Nature (London), 185, 534 (1960).

5) see for example K. Kakemi, H. Sezalki, and K. Ikeda, Yakuzaigaku, 28, 101 (1968); E. M. Sellers and J. Koch-Weser, Ann. N.Y. Acad. Sci., 179, 213 (1971). 
not made satisfactorily. In the great majority of cases, drugs are presumed to interact with macromolecular components of tissues. The interaction arises from the concerted operation of numerous bond of several types, i.e. covalent bonds, hydrogen bonds, ionic bonds, and hydrophobic bonds etc. Therefore, it is helpful to understanding of the action of drugs that effect of drugs on hydrophobic binding is defined. In this investigation, we used aqueous solutions of surfactant micelles as a simple model system containing hydrophobic bonds and assessed relatively easily the hydrophobic bonds through measurement of the critical micelle concentration $(\mathrm{cmc}){ }^{6)}$ The particular surfactants chosen were sodium dodecyl sulfate (SDS) and $n$-dodecyl-N-dimethylethylammonium bromide (DDAB), and their $\mathrm{cmc}^{\prime}$ s were measured in the presence of drugs, i.e. pyrazolone derivatives which were analgesic and antipyretic.

\section{Experimental}

Materials-Antipyrine (Iwaki Seiyaku Co.) and 4-aminoantipyrine (Wako Pure Chemical Ind.) were used and recrystallized from hot benzene. The recrystallized products had melting ranges of $112-113^{\circ}$ and $108-109^{\circ}$, respectively. Phenylbutazone was obtained from Fujisawa Pharmaceutical Co., Itd. and recrystallized from methanol $\left(\mathrm{mp} 104-105^{\circ}\right)$. The SDS crystals (Wako Pure Chemical Ind.) were recrystallized from $95 \%$ ethanol according to the method of Emerson.7) The recrystallization procedure was repeated (2-3 times) until the $\mathrm{cmc}$, the equivalent conductance at $\mathrm{cmc}$, and the equivalent conductance at infinite dilution of the product in water at $25.0^{\circ}$ were in close agreement with the accepted values for SDS. ${ }^{8)}$ The DDAB (E. Merck, Darmstdt) was recrystallized from acetone-ether twice.

Conductivity Measurements-Surfactants and drug were dissolved in distilled and deionized water, and these solutions were adjusted to volume in calibrated flasks at $25.0^{\circ}$. The $\mathrm{pH}$ values of the solutions became 5.1. Conductivities of surfactant solutions containing drugs were measured using a conduct meter (CM-1DB type, TOA Denpa Co.) at $25.0^{\circ}$ according to the method of Adderson et al. ${ }^{9}$ (For each sample $\mathrm{cmc}$ values were obtained from the point of intersection of the two extrapolated straightline portions of the specific conductance versus concentration graph. The cmc values for each sample agreed within the limits of experimental error $( \pm 2.0 \%)$.

Solubility Determination-To measure the cmc of the surfactants in the presence of phenylbutazone equilibrium solubility of the drug in various concentration of surfactant solution was determined at $25.0^{\circ}$. After equilibrium, sample solutions were removed by syringe, and filtered through the membrane (Sartorius Membrane Filter SM 11307, pore size $0.2 \mu \mathrm{m}$ ) quickly. The samples were assayed at $260 \mathrm{~nm}$ using Hitachi Type 100-20 spectrophotometer. No significant absorbance was found for the surfactant at $260 \mathrm{~nm}$ for the drug analysis.

\section{Results and Discussion}

The conductivity critical micelle concentrations $(\mathrm{cmc})$ of SDS and DDAB in 0.5 м antipyrine and $0.5 \mathrm{~m} 4$-aminoantipyrine solutions were measured at $25.0^{\circ}$. Some typical plots of the conductance data from which the $\mathrm{cmc}^{\prime}$ s were derived are presented in Fig. 1 and the cmc's obtained by conductivity measurements are shown in Table I.

The results in pure water agree with those of other investigators, ${ }^{8)}$ and the cmc values in the presence of antipyrine or 4-aminoantipyrine were lager than those obtained without the drugs. The variation of the $\mathrm{cmc}$ in the presence of the drug is similar to that observed for other surfactant system with additives, i.e. urea ${ }^{6,10)}$ and glucose. ${ }^{11)}$ Thus, antipyrine and 4-aminoantipyrine do break hydrophobic bonds in aqueous solutions. These drugs are not as effective in breaking up SDS micelles as they are in breaking up DDAB micelles. Antipyrine raises the cmc of DDAB by $9.8 \%$ and 4 -aminoantipyrine raises it by $2.3 \%$; the corresponding

6) M.F. Emerson and A. Holtzer, J. Phys, Chem., 71, 3320 (1967).

7) M.F. Emerson, ph.D. Thesis, Washington University, St. Louis, Mo., 1966.

8) A. Wilson, M. Epstein, and J. Ross, J. Colloid Sci., 12, 345 (1957); P. Mukerjee, K.J. Mysels, and C.I. Dulin, J. Phys. Chem., 62, 1390 (1958).

9) J.E. Adderson and H. Taylor, J. Colloid Sci., 19, 495 (1964).

10) W. Bruning and A. Holtzer, J. Am. Chem. Soc., 83, 4865 (1961).

11) J.E. Adderson and C.G. Butler, J. Pharm. Pharmacol., 24, 130 (1972). 


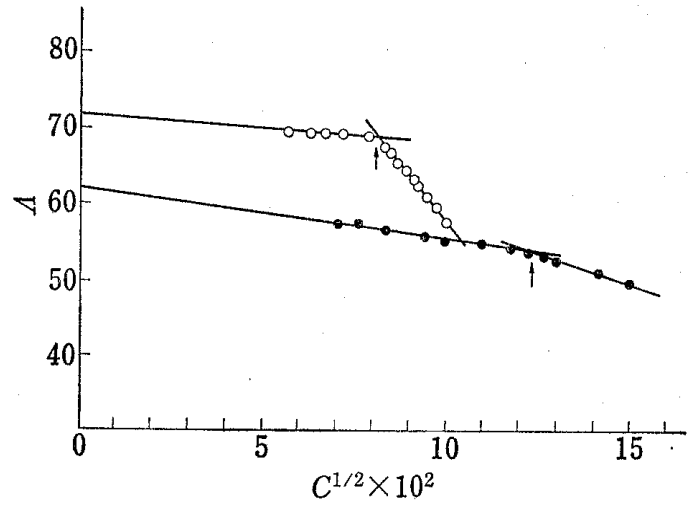

Fig. 1. Conductometric Curves for SDS at $25.0^{\circ}$

$\mathrm{O} ; \mathrm{H}_{2} \mathrm{O}$, ; 0.5м 4-aminoantipyrine, $\uparrow$; cmc. Concentration $(C)$ is in mol per liter.

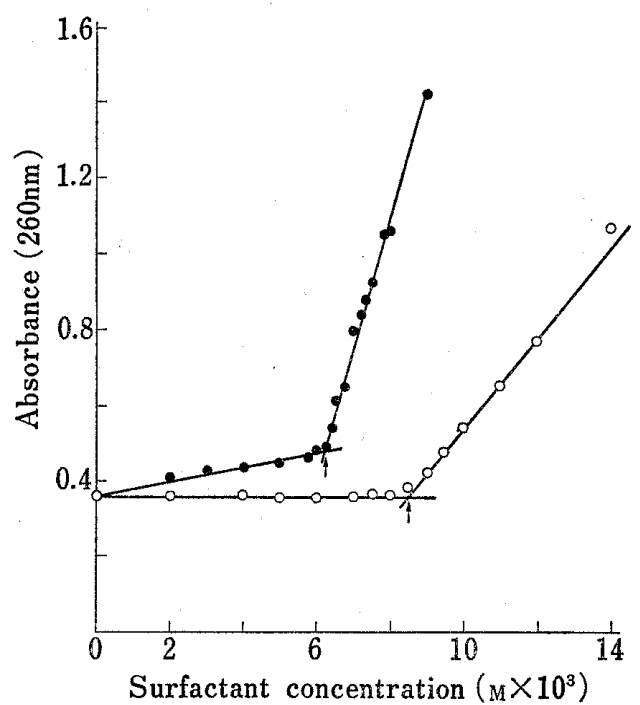

Fig. 2. Effect of SDS and DDAB on Solubility of Phenylbutazone in Water at $25.0^{\circ}$

; SDS, O; DDAB, $\uparrow$; cmc.

TABLE I. Critical Micelle Concentration for SDS and $\mathrm{DDAB}$ in Additive Solution at $25.0^{\circ} a$ )

\begin{tabular}{cccc}
\hline Surfactant & 0 & $0.5 \mathrm{M}$ antipyrine & $0.5 \mathrm{M}$ 4-aminoantipyrine \\
\hline SDS & $7.65 \times 10^{-3}$ & $1.26 \times 10^{-2}$ & $1.45 \times 10^{-2}$ \\
DDAB & $1.33 \times 10^{-2}$ & $1.46 \times 10^{-2}$ & $1.36 \times 10^{-2}$ \\
\hline
\end{tabular}

a) These values were determined by conductivity measurements cmc's are in mol per liter.

values for SDS are $64.7 \%$ and $89.5 \%$. Antipyrine and 4-aminoantipyrine are basic drugs and $\mathrm{p} K_{\mathrm{a}}$ values of the drugs are 1.4 and 4.4, respectively. Under the experimental conditions $(\mathrm{pH} \mathrm{5.1)}$, antipyrine is nonionized and $16.6 \%$ of the molecules of 4 -aminoantipyrine are in ionized form. If the drugs decrease the repulsive force between the ionic heads of the surfactant molecules, the stability of micelles may increase. Since the drugs used are basic substances, it is not expected that the drugs decrease the repulsive force between the cationic heads of DDAB. However, the stability of SDS micelles rather than DDAB micelles decreased by addition of the drugs to the surfactant solutions. It is presumed from these results that the effect of the drugs on the intermolecular interactions of the surfactants dose not contribute to the stability of the micelles, but we can't give the rational interpretation for the relation between ionic interaction and micelle stability in these systems.

The dielectric constant of $0.5 \mathrm{~m}$ antipyrine solution is nearly equal to that of pure water at $25.0^{\circ}{ }^{12}$ This fact indicates that the average dielectric constant of the medium is not the determining factor of destorying micelles. Since antipyrine and 4-aminoantipyrine are very water soluble, ${ }^{13)}$ these drug molecules may have strong affinity for water molecules. This affinity of the drug for water maybe alter the water structures. That is, the "ice-berg" structures will be modified and hydrophobic interaction will be reduced.

Micelles of SDS or DDAB is unstable in the presence of antipyrine and 4-aminoantipyrine but the addition of phenylbutazone results in increase in stability of micelles (Fig. 2).

12) The dielectric constant of $0.5 \mathrm{~m}$ antipyrine was determined by measuring the electric capacitance of two metal plates set into glass ressel and insulated from each other. These apparatus was made by hand in other laboratory.

13) Y. Morimoto, R. Hori, and T. Arita, Chem. Pharm. Bull. (Tokyo), 22, 2217 (1974). 
The cmc's of SDS and DDAB fell from $7.65 \times 10^{-3} \mathrm{M}$ to $6.23 \times 10^{-3} \mathrm{M}(19 \%)$ and from $1.35 \times 10^{-2} \mathrm{M}$ to $8.50 \times 10^{-3} \mathrm{M}(36 \%)$, respectively. The cmc values were determined by measuring the solubility of phenylbutazone in the presence of the surfactants since the drug is very slightly soluble in water $\left(0.7 \mathrm{mg} / \mathrm{ml}\right.$ at $\left.22.5^{\circ}\right) .{ }^{14)}$ Solubilization is, of course, closely related to micellization since little or no solubility increase is observed until the cmc of the surfactant is reached, but once the micelles are fully formed its increase is directly proportional to the concentration of the surfactant over a large range. Some mechanisms of the solubilization in aqueous micellar solution were shown by Fendler ${ }^{15)}$ et al. but quantitative description of the mechanism of the solubilization is available in this experiment.

Antipyrine and 4-aminoantipyrine which are very water soluble are in breaking up SDS or DDAB micelles. Phenylbutazone which is very slightly soluble in water is in no breaking up their micelles. The mode of action of these pyrazolone derivatives could not be proposed now, but it is presumed that the affinity of these drugs for water plays an important role in stability of micelles. Spelling out the details of the relation between hydrophobic interaction and pyrazolone derivatives will have to await further elucidation of the effect of these drugs on the structure of water.

14) M. Windholz, S. Budavari, L.Y. Stroumtsos, and M.N. Fertig, "The Merck Index," Merck and Co., Inc., Rahway, 1976, p. 964.

15) E.J. Fendler and J.H. Fendler, Adv. Phys. Org. Chem., 8, 271 (1970). 